\title{
Direct control of transcription of the Drosophila morphogen bicoid by the Serendipity $\delta$ zinc finger protein, as revealed by in vivo analysis of a finger swap
}

\author{
François Payre, Michèle Crozatier, and Alain Vincent ${ }^{1}$ \\ Centre de Biologie du Développement, Unité Mixte de Recherche (UMR) 9925 du Centre National de le Recherche \\ Scientifique (CNRS), Université Paul Sabatier, 31062 Toulouse Cedex, France
}

\begin{abstract}
Determination of the anterior structures of the Drosophila embryo is under control of the maternal gene product Bicoid $(b c d)$, which specifies distinct domains of embryonic gene expression in a concentration-dependent manner. We show here that bcd transcription is controlled by serendipity $\delta$ (sry $\delta$ ), a zygotic-lethal zinc finger protein gene. This sry $\delta$ germ-line function was revealed by transgenic expression of a modified Sry $\delta$ protein, Sry DB56, carrying a two-finger swap. Although it almost fully rescues sry $\delta$ lethality, Sry DB56 does not substitute for the wild-type protein in activating bcd transcription. Two overlapping sites binding the Sry $\delta$ protein were identified in the bcd promoter region, a few base pairs upstream of the putative TATA box. Mutating one site impairs bcd transcription in vivo, indicating that Sry $\delta$ acts directly upstream of $b c d$. The specific requirement of $s r y \delta$ for bcd transcription in the female germ line constitutes an unexpected link between a zygotic gene with pleiotropic functions and the establishment of coordinates of the Drosophila egg. It highlights the fundamental role of ubiquitous transcription factors in bringing about a specific developmental program.
\end{abstract}

[Key Words: Drosophila; bicoid; serendipity; transcription; zinc finger]

Received August 8, 1994; revised version accepted September 26, 1994.

In Drosophila, combined genetic and molecular analyses have defined an elaborate cascade of transcription factors that controls the basic organization and polarity of the two major axes, anteroposterior and dorsoventral, of the embryo (for review, see St Johnson and Nüsslein-Volhard 1992; Hoch and Jäckle 1993). Elaboration of the body plan in the head and thoracic regions is largely controlled by the anterior patterning system (Nüsslein-Volhard et al. 1987). Four maternal-effect genes, bicoid (bcd), exuperentia (exu), swallow (swa) and staufen (stau), are genetically identified components of this anterior system (Schüpbach and Wieschaus 1986; Frohnhöfer and Nüsslein-Volhard 1987; St Johnson et al. 1989), with bcd being the decisive component (Frohnhöfer and NüssleinVolhard 1986; Driever et al. 1990). The relative severity of cuticular phenotypes associated with different $b c d$ mutations defines an allelic series. Weak alleles affect the anterior-most structures of the head, the labral derivates, whereas strong alleles develop neither head nor thorax and carry a duplication of the posterior-most

${ }^{1}$ Corresponding author. structure, the telson, at the anterior end. Activity of exu, $s w w$ and stau is required to localize bcd RNA to the anterior pole of the egg during oogenesis. Localized $b c d$ RNA is translated after fertilization to give rise to an anteroposterior gradient of the protein that peaks at the anterior pole (Driever and Nüsslein-Volhard 1988a). Bicoid has all the properties of a morphogen, that is, it determines cell fate along the anteroposterior axis of the embryo in a concentration-dependent manner (Driever and Nüsslein-Volhard 1988b). The Bcd protein contains a homeo domain and is thought to primarily activate transcription of different zygotic head and thoracic segmentation genes in specific domains of the embryonic head and trunk regions (Tautz 1988; Driever and NüssleinVolhard 1989; Struhl et al. 1989; Finkelstein and Perrimon 1990; Hoch et al. 1991; Pignoni et al. 1992; for review, see Driever 1992). Down-regulation of Bcd activity at the anterior pole has recently been shown to be under the control of the torso receptor-mediated signaling pathway (Ronchi et al. 1993).

A series of elegant experiments by Driever and Nüsslein-Volhard (1988b) and Struhl et al. (1989) has demonstrated that (1) for the anterior pattern of segmenta- 
tion, cell position information is largely determined by the local concentration of Bcd protein, and (2) the maximum concentration of Bcd protein is roughly proportional to the number of $b c d$ gene copies. Together, these data suggest that the level of Bcd activity in early embryos reflects the level of $b c d$ transcription. However, nothing is known of the transcriptional control of $b c d$.

We report here that serendipity delta (sry $\delta$ ), which encodes a transcriptional activator of the $\mathrm{C} 2 \mathrm{H} 2$ zinc finger type, is required for $b c d$ transcription. Sry $\delta$ was initially characterized as a zygotic lethal gene with pleiotropic mutation effects; single amino acid changes in different domains of the Sry $\delta$ protein caused embryonic and sex-biased lethality. Both somatic and germ-line defects are associated with sry $\delta$ mutations, the relative severity of which depends on the allele considered (Crozatier et al. 1992). Whereas a single copy of wild-type sry $\delta$ fully suppresses sry $\delta$ mutant lethality and sterility, increasing the copy number of sry $\beta$, a sry $\delta$ paralog (Vincent et al. 1985; Ferrer et al. 1994), does not rescue any of these phenotypes (Crozatier et al. 1992). Both Sry $\beta$ and Sry $\delta$ are sequence-specific DNA-binding proteins that bind in vivo to distinct sets of specific sites on polytene chromosomes (Payre and Vincent 1991; Noselli et al. 1992). In vivo experiments in which the six contiguous zinc finger domains were reciprocally swapped between Sry $\beta$ and Sry $\delta$ indicated that the specificity of chromosomal binding of each protein is largely, although not exclusively, dependent on DNA-specific recognition (Noselli et al. 1992). To gain further insight into the contribution of separate zinc fingers to the functional difference between Sry $\beta$ and Sry $\delta$, as well as to identify some of their respective gene targets, we expressed modified Sry $\delta$ proteins containing chimeric Sry $\beta / \delta$ zinc finger domains. One construct, sry DB56, that encodes a Sry $\delta$ protein in which fingers 5 and 6 are replaced by the homologous fingers of Sry $\beta$, fully suppressed sry $\delta$ lethality but brought to light one maternal effect of sry $\delta$ mutations; embryos laid by sry DB56-rescued females do not hatch and display a $b c d$-like phenotype suggesting a misexpression of $b c d$. Our data show that Sry $\delta$ controls $b c d$ activity at the transcriptional level, by binding to a cisacting element within the $b c d$ promoter. Identification of Sry $\delta$ as a component in regulation of $b c d$ expression adds yet another step to the cascade of transcriptional regulatory interactions establishing the polarity of the anteroposterior axis of the Drosophila embryo. The specific requirement of sry $\delta$ for $b c d$ transcription in female germ-line cells illustrates how fundamental the role of ubiquitous regulatory factors can be in bringing about a specific developmental program.

\section{Results}

Swapping fingers 5 and 6 between the Sry $\delta$ and Sry $\beta$ proteins; in vitro binding studies

Previously, we reported the respective DNA recognition sequences of the closely related Drosophila Sry $\beta$ and Sry $\delta \mathrm{C} 2 \mathrm{H} 2$ zinc finger proteins, 5'-YCAGAGATGCGCA-3' and 5'-YTAGAGATGGRAA-3', respectively (Payre and Vincent 1991). The Sry $\beta$ and Sry $\delta$ consensus binding sites, therefore, differ at 4 of the 13 nucleotide positions (Fig. 1A), and these differences are sufficient for discriminatory binding of Sry $\beta$ and Sry $\delta$ to their respective in vitro recognition sites (F. Payre, unpubl.). The DNAbinding specificity of Sry $\beta$ and Sry $\delta$ is dictated in vitro only by the domain of six adjacent zinc fingers (Noselli et al. 1992). Amino acid sequence comparison of the two proteins reveals that fingers 2,3 , and 4 are very similar, whereas fingers 1,5 , and 6 are much more divergent (Vincent et al. 1985; Payre et al. 1990). To test for the specific contribution of fingers 5 and 6 in selective binding of Sry

A

A

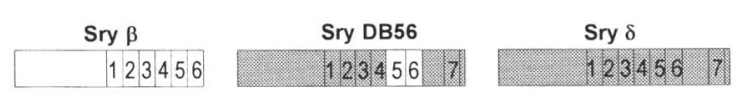

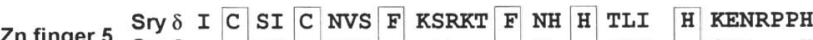

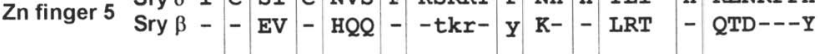

\begin{tabular}{llll|l|l|l|l|l|l|l|l|l|l|l} 
Zn finger 6 & Sry $\delta$ & Y & C & SV & C & PKS & F & TERYT & L & KM & H & MKT & H & EGDVVTG
\end{tabular} Sry $\beta$ P - PD - E-- - Vdk-- - $-\mathrm{V}$ H KrVQ - QPVEKP-

consensus binding site:

Sry $\beta: \quad 5^{\prime}$ YAGAGATG CGCA $3^{\prime}$

Sry $\delta:$ 5' TAGAGATG GRAA 3'

B

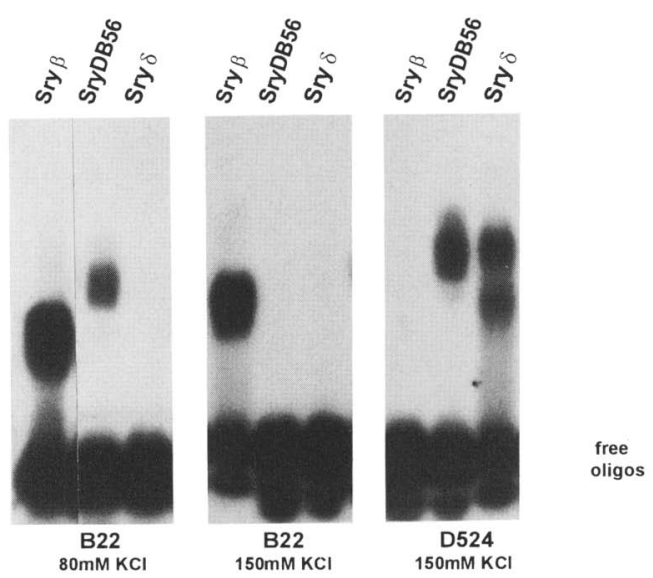

Figure 1. DNA-binding properties of the Sry DB56 protein. $(A)$ Schematic representation of the Sry DB56 protein. Zinc fingers 5 and 6 of Sry $\delta$, right (shaded boxes), have been replaced by the corresponding fingers of Sry $\beta$, left (open box). This selective finger swap results in 36 of 58 amino acid changes, with 7 of these changes being conservative $(E \rightarrow D),(S \rightarrow T),(K \rightarrow R)$. The 13-bp consensus recognition sites of Sry $\beta$ and Sry $\delta$ are aligned with nucleotide differences indicated by italics. $(B)$ EMSA of DNA recognition by the Sry DB56 protein. Purified proteins made in $E$. coli were incubated with oligonucleotides containing either a Sry $\beta$ (B22) or Sry $\delta$ (D524) consensus DNA-binding site. Using moderate ionic strength conditions (150 mM KCl) Sry DB56 DNA-binding properties are indistinguishable from Sry $\delta$, except for the relative migration of the formed complex. In lower ionic strength conditions $(80 \mathrm{mM})$ Sry DB56 binds also to the oligonucleotide containing a Sry $\beta$ consensus-binding site. 
$\beta$ and Sry $\delta$ to their respective recognition sequences, we examined the binding specificity of a chimeric Sry $\delta$ protein, Sry DB56, in which fingers 5 and 6 were exchanged for the corresponding domains of Sry $\beta$ (Fig. 1A). The Sry $\beta$, Sry $\delta$, and Sry DB56 proteins, purified from overproducing Escherichia coli strains, were assayed for specific binding to oligonucleotides containing either a Sry $\beta$ (B22) or a Sry $\delta$ (D524) consensus binding site, in electrophoretic mobility shift assays (EMSAs). Compared with wild-type Sry $\delta$, Sry DB56 does not display a major modification of its DNA recognition properties in vitro, as it binds efficiently to the Sry $\delta$-binding site under all experimental conditions tested (Fig. 1B). The fact that at low ionic strength Sry DB56 also binds to the Sry $\beta$-binding site suggests, however, a more relaxed binding specificity.

\section{The chimeric SryBD56 protein reveals a maternal sterility effect of sry $\delta$ mutations}

To test for the functional consequences in vivo of swapping fingers 5 and 6 between Sry $\beta$ and Sry $\delta$, we constructed transgenic lines expressing the Sry BD56 chimeric protein under the control of the sry $\delta$ promoter and assayed for its ability to rescue sry $\delta$ lethal mutations. When the $\mathrm{p}$ [sry DB56] transposon was introduced into sry $\delta^{-}$hemizygotes there was almost complete rescue of lethality, for each of the four sry $\delta$ alleles (Table 1). Similar results were obtained using two independent insertions on different chromosomes (Table 1 ; data not shown). In all cases, the rescued males were fully fertile. Strikingly, however, the rescued females mated to wildtype males do not give any viable progeny, although egg laying is normal. This correlates with the normal morphology of ovaries from rescued females upon visual inspection by light microscopy (data not shown). Therefore, sry DB56 behaves like a maternal effect-specific allele of sry $\delta$, thus providing access to at least some sry $\delta$ maternal functions.

\section{Maternal phenotype of sry $\delta$ : bcd-like}

Embryos laid by sry $\delta$ mutant females rescued by introduction of p[sry DB56] develop to the point of making cuticle. The cuticular pattern of the embryos (Fig. 2) shows reductions and/or deletions of distinct anterior structures, depending on the sry $\delta$ allele. In the case of the sry $\delta^{S F 1}$ allele, only the anterior-most head structure, the labrum, is affected. In the case of sry $\delta^{12}$, all derivatives of the head are affected. In the case of the $s r y \delta^{S F 2}$ or sry $\delta^{14}$ alleles, thoracic segments are missing and elements of the posterior telson are duplicated at the anterior (Fig. 2). In the strongest sry $\delta$ allele, sry $\delta^{14}$ (Crozatier et al. 1992), segmentation defects are also observed frequently in the anterior abdomen, most often in the abdominal segments A2, A3, or A4. The fact that similar results are obtained with the p[sry $B D 56]$ transposon inserted on the two different chromosomes confirms that the observed phenotype is not attributable to an insertion position effect (Table 1; data not shown). The phe-
Table 1. Rescue of sry $\delta$ alleles by one copy of the chimeric sry DB56 gene

\begin{tabular}{lcccc}
\hline $\begin{array}{l}\text { sry } \delta \\
\text { alleles }\end{array}$ & $\delta^{12}$ & $\delta^{14}$ & $\delta^{\text {SF1 }}$ & $\delta^{\text {SF2 }}$ \\
\hline$\Sigma$ & 1367 & 837 & 1217 & 1550 \\
$S b / 4^{\mathrm{a}}$ & 256 & 146 & 194 & 254 \\
F non-Sb & 207 & 138 & 237 & 287 \\
M non-Sb & 184 & 143 & 240 & 295 \\
VaF\% & 81 & 95 & 122 & 113 \\
$\quad(\mathrm{~F})$ & $(0)$ & $(0)$ & $(0.4)$ & $(1.5)$ \\
VaM\% & 72 & 98 & 124 & 116 \\
$(\mathrm{M})$ & $(0.4)$ & $(0.5)$ & $(30)$ & $(80)$ \\
\hline
\end{tabular}

Data are for a transgenic line with the $\mathrm{p}\left[\right.$ sry $\left.D B 56 ; r^{+}{ }^{+}\right]$transposon inserted on the $\mathrm{X}$ chromosome and introduced into sry $\delta$ hemizygous backgrounds using the following cross:

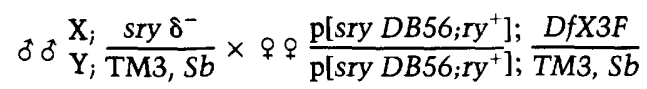

Non-Stubble ( $S b$, a dominant marker of bristles) females and males have received one copy of the transposon and correspond to rescued adults. Viability indexes (noted $\mathrm{VaF} \%$ and $\mathrm{VaM} \%$ for females and males, respectively/ were calculated as follows:

$$
\mathrm{Va} \%=\frac{\text { non }-S b}{S b / 4^{\mathrm{a}}} \times 100
$$

Below, and in parenthesis, is the percentage of viable escaper adults observed in the same crosses but in the absence of the $\mathrm{p}$ [sry DB56] transposon (Crozatier et al. 1992). Viability obtained with the $\mathrm{p}\left[\right.$ sry $\left.D B 56 ; \mathrm{ry}^{+}\right]$transposon on the second chromosome was $100 \%$ in the case of the sry $\delta^{\mathrm{SF} 2}$ and $\delta^{14}$ alleles, $87 \%$ and $100 \%$ for females and males, respectively, for the sry $\delta^{12}$ allele, and not determined for the sry $\delta^{\mathrm{SF} 1}$ allele.

aThe viability of $S b$ flies is lower than wild-type flies. This difference is taken into account in our calculations by introducing a correction coefficient of 1.05 , as determined in control experiments (data not shown).

notype of embryos laid by the p[sry $B D 56]$ rescued sry $\delta^{-}$ females is clearly reminiscent of that described for a $b c d$ hypomorphic series (Fronhöfer and Nüsslein-Volhard 1987). These results raised the possibility that wild-type sry $\delta$ function is necessary for normal levels of $b c d$ activity. This hypothesis was supported by the progressively stronger shift toward the anterior in the positions of the cephalic furrow and the anterior margin of Krüppel expression that is observed in embryos, when going from weak to strong sry $\delta$ mutant alleles (data not shown). Such a shift in the fate map of the early embryo is diagnostic of a deviation from the normal dose of $b c d^{+}$activity (Driever and Nüsslein-Volhard 1988). That the $b c d$-like phenotype is not attributable to a dominantnegative effect of the chimeric gene was further confirmed by examining embryos laid by the very few transallelic female escapers that emerge in the sry $\delta^{S F 1} /$ sry $\delta^{S F 2}$ combination. The cuticular phenotype of these embryos is indistinguishable from that of strong $b c d \mathrm{mu}-$ tant embryos (Fig. 2). Together, these results suggest that 


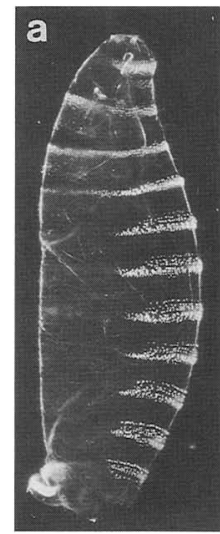

P[sry DB56]; $/ /+$

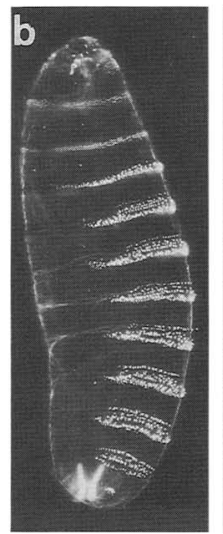

P[sry DB56]; ; - \&SF1

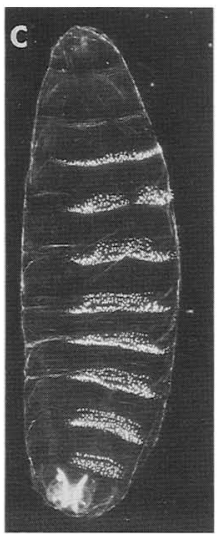

$p[s r y D B 56] ;-/ \delta^{12}$

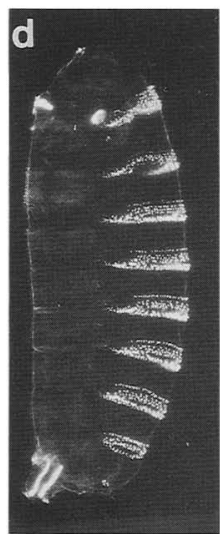

p[SryDB56]; ; - $S$ SF2

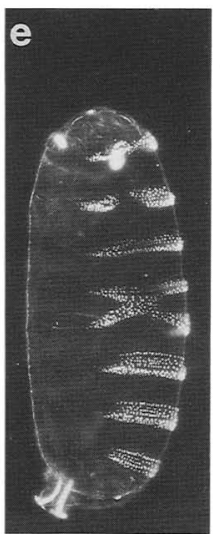

$P[$ sry $D B 56] ;-/ \delta^{14}$

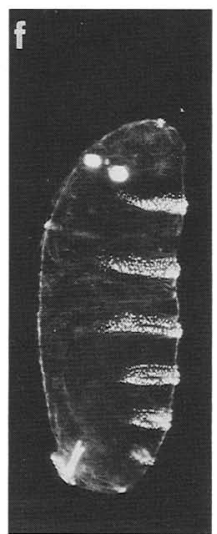

${ }_{\delta} \mathrm{SF} / \delta \mathrm{SF2}$

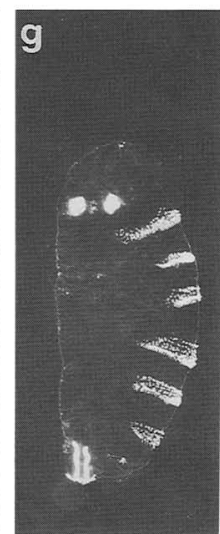

$b c d^{6}$

Figure 2. $b c d$, a maternal effect of sry $\delta$ mutations revealed in p[sry $D B 56]$ rescue experiments. Cuticle preparations of wild-type and sry $\delta$ mutant, compared to $b c d$ embryos; anterior is at the top in all cases. $(a) \mathrm{p}[$ sry $D B 56] ; D f X 3 F-/+$. Cuticle is wild type. $(b-e) \mathrm{p}[$ sry $D B 56] ; D f X 3 F /$ sry $\delta^{-} ;(b) \delta^{\mathrm{SF} 1},(c) \delta^{12},(d) \delta^{\mathrm{SF} 2},(e) \delta^{14}$. Note the gradually more severe anterior defects shown by sry $\delta$ mutant embryos going from a weak $\left(\right.$ sry $\left.\delta^{\mathrm{SF} 1}\right)$ to a strong $\left(\right.$ sry $\left.\delta^{14}\right)$ sry $\delta$ allele. $(f)$ embryos laid by sry $\delta^{\mathrm{SF} 1} /$ sry $\delta^{\mathrm{SF} 2}$ transheterozygote escaper females. In these embryos, as for embryos laid by p[sry $D B 56] ; D f X 3 F / \delta^{14}$ females head and thorax are replaced by a duplicated telson and one to several abdominal segments are missing, a phenotype clearly reminiscent of the strong $b c d^{6}$ mutation $(g)$.

one maternal function of sry $\delta$ is to positively regulate the level of bcd activity.

\section{sry $\delta$ controls the level of bcd $m R N A$} accumulation in oocytes

To test whether the reduction of bcd activity in sry $\delta$ mutant embryos is attributable to a decreased level of $b c d$ transcription, we prepared RNA from ovaries of sry $\delta$ mutant females rescued by the presence of the p[sry $D B 56$ ] transposon, sry $\delta^{S F 2}$ homozygous females (only for this allele, sterile adult females emerge), and some rare sry $\delta^{S F 1} /$ sry $\delta^{S F 2}$ and sry $\delta^{12} /$ sry $\delta^{S F 2}$ transheterozygote escaper females. Northern blot analysis shows that mutations in sry $\delta$ drastically decrease the level of $b c d$ RNA accumulation, which is undetectable in the strong sry $\delta$ allelic combinations (Fig. 3A). This reduction of mRNA level appears to be specific to $b c d$, as other genes tested, either strictly maternal such as nanos (Wang and Lehmann 1991) and oskar or housekeeping (rp49, rpL17A), are unaffected in their mRNA levels (Fig. 3; data not shown). Quantitation of the level of bcd mRNA in different sry $\delta$ mutant backgrounds (indicated below the blot) shows that sry $\delta$ controls $b c d$ expression at the level of RNA accumulation. The Northern blot data also indicate that the level of $b c d$ RNA in females carrying four copies of $b c d$ is $\sim 1.7$ times that of the wild type. This is independent of the copy number, 1 or 2, of the wild-type sry $\delta$ gene, showing that for $b c d$ transcription Sry $\delta$ is not a limiting factor. Low amounts of bcd mRNA are detected in ovaries of the p[sry DB56]; sry $\delta^{S F 1}$ or $\delta^{12} \mathrm{mu}-$ tant females (Fig. 3A). In situ hybridization on wholemount ovary preparations show that this mRNA is normally localized at the anterior pole of the growing oocyte (Fig. 3B), indicating that sry $\delta$ mutations do not interfere with $b c d$ RNA prelocalization. Furthermore, the pres- ence and normal localization of oskar RNA (Ephrussi et al. 1991) in ovaries of $\mathrm{p}$ [sry DB56]-rescued sry $\delta^{14}$ mutant females show that the absence of detectable $b c d$ mRNA is not attributable to a general effect of sry $\delta$ mutations on synthesis, transport, and/or localization of mRNA in oocytes via, for example, general defects of membrane or cytoskeletal elements (Theurkauf et al. 1992). Therefore, we conclude that sry $\delta$ is specifically required for $b c d$ transcription during oogenesis.

\section{Wild-type sry $\delta$ activity in germ-line cells is required for proper oogenesis in addition to bcd transcription}

Whereas $b c d$ transcription is restricted in the ovary to the germ line, the Sry $\delta$ protein is present in nuclei of both the germ line (nurse cells) and somatic (follicle cells) components of the ovary (Payre et al. 1989). To test the germ-line effect of sry $\delta$ mutations, we generated homozygous sry $\delta$ germ-line clones by X-ray-induced $\mathrm{mi}$ totic recombination in heterozygous sry $\delta^{-}$females carrying, in trans, a transposon $\mathrm{p}\left[\mathrm{ovO}^{D 1}-8\right]$ (Mevel-Ninio et al. 1994; see Materials and methods). Because of the dominant effect of $O V O^{D 1}$, a block of oogenesis at previtellogenic stages, developed ovarioles found in irradiated sry $\delta^{-} / \mathrm{p}\left[0 v o^{D 1} \cdot 8\right]$ females derive only from sry $\delta$ homozygous mutant germ-line cells generated by recombination. Examination of these ovaries, using differential interference contrast (DIC) optics and fluorescent probes for actin and DNA, revealed profound alterations in the cellular morphology of sry $\delta^{-}$ovarioles, starting at midoogenesis (data not shown). In no case did we observe significant egg deposition. Furthermore, no Bcd protein is detected in the few embryos derived from sry $\delta^{-}$homozygous germ-line clones (data not shown), confirming the requirement of Sry $\delta$ for $b c d$ transcription. 
Figure 3. Defective accumulation of $b c d$ mRNA in sry $\delta$ mutant ovaries. $(A)$ Northern analysis of $b c d$ expression in ovaries of sry $\delta$ mutant females. Fifteen micrograms of total RNA extracted from hand-dissected ovaries was run in each lane of a formaldehyde-agarose gel, transfered to a nitrocellulose membrane, and probed with labeled bcd cDNA (Berleth et al. 1988) together with rp49 (O'Connell and Rosbash 1984). A single $b c d$ transcript of $\sim 2.6 \mathrm{~kb}$ was detected. The relative level of $b c d$ mRNA accumulation in ovaries of wild-type flies, flies containing four copies of $b c d$ (Struhl et al. 1989], and p[sry BD56] containing flies in the context of different sry $\delta$ mutant combinations was calculated using rp49 and rpL17A (Noselli and Vincent 1992) as internal standards for quantitation of deposited RNA, and is indicated below each lane. Wild-type levels of $b c d$ mRNA are observed with a single copy of sry $\delta$, in the presence or absence of p[sry DB56]. Low amounts of $b c d$ mRNA are detected in ovaries of sry $\delta^{\mathrm{SF} 1}$ and sry $\delta^{12}$ hemizygous or sry $\delta^{\mathrm{SF} 1} /$ sry $\delta^{\mathrm{SF} 2}$ transheterozygote females, and no signal can be observed in ovaries from sry $\delta^{\mathrm{SF} 2}$ and sry $\delta^{14}$ hemizygous or sry $\delta^{\mathrm{SF} 2}$ homozygous flies or sry $\delta^{\mathrm{SF} 2} /$ sry $\delta^{12}$ transheterozygote escaper females. $(B)$ Detection of $b c d$ mRNA in whole-mount egg chambers by in situ hybridization using a digoxigenin-labeled probe. (a) p[sry DB56]; $D f X 3 F /+$ egg chambers. Similar results were obtained with wild-type ovaries. $(b)$ $\mathrm{p}[$ sry $D B 56] ; D f X 3 F /$ sry $\delta^{\mathrm{SF} 1},(c)$ sry $\delta^{12}$, and (d) sry $\delta^{14}$ egg chambers. (d) Small amounts of an oskar DNA probe (Ephrussi et al. 1991) have been added to the hybridization mixture. In all panels, anterior is to the left and posterior to the right. Arrowheads indicate the normal anterior localization of the low amounts of $b c d$ mRNA detected in sry $\delta^{\mathrm{SF} 1}$ and sry $\delta^{12}$ mutant ovaries at stage $10 \mathrm{~A}$ of oogenesis. No signal corresponding to $b c d$ mRNA can be detected at any stage in sry $\delta^{14}$ mutant egg chambers.
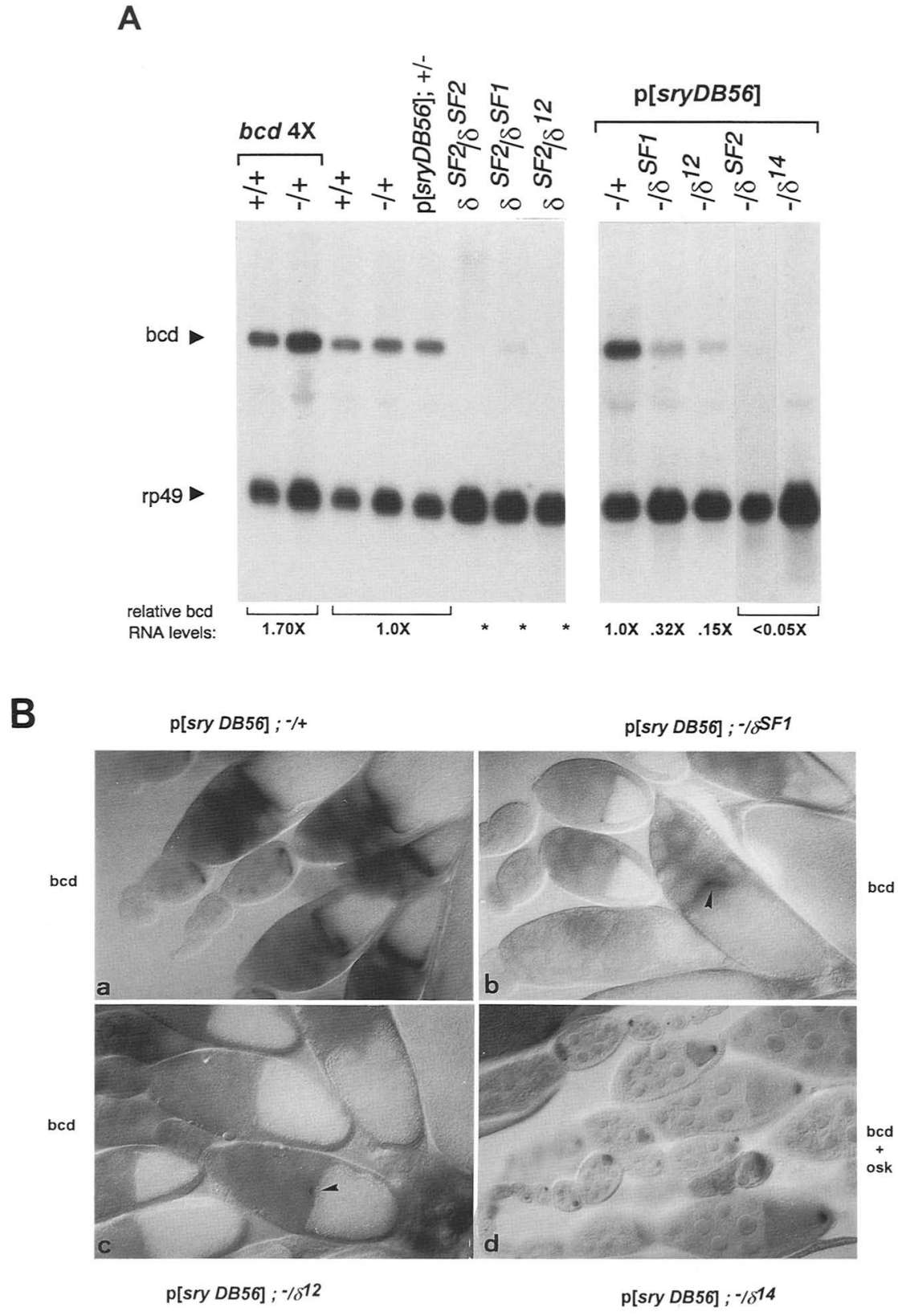

Sry $\delta$ binds in vitro to bcd DNA, upstream of the putative TATA box

As a first step toward testing for a possible direct interaction between Sry $\delta$ and $b c d$, we looked for in vitro binding sites of the Sry $\delta$ protein within the $b c d$ genomic region shown previously to fully rescue the $b c d$ phenotype. Three plasmids containing $b c d$ DNA were used for immunoprecipitation with the Sry $\delta$ protein. A single fragment was precipitated with DNA cut with different restriction enzymes, locating the Sry $\delta$-binding site close upstream of the $b c d$ transcription start. Immunoprecipitation analysis performed in parallel using the Sry DB56 protein indicated that the chimeric protein is able to bind to the same DNA fragment, and with an efficiency similar to that of wild-type Sry $\delta$ (Fig. 5B). Further mapping allowed the Sry $\delta$-binding site to be located within a 70-bp fragment containing the putative $b c d$ TATA box (Figs. 4 and 5A). The nucleotide sequence of this fragment revealed the presence of two partly overlapping motifs displaying extensive sequence homology with the Sry $\delta$ consensus DNA-binding site (Fig. 5A; see Fig. 1A). Point mutations were introduced at either of these two sites. In both cases (N1 and N3 mutations respectively), binding of Sry $\delta$ to the $b c d$ promoter region is drastically decreased (Fig. 5B), whereas elimination of both sites, using a 12-bp deletion (NO mutation), fully abolishes Sry $\delta$ binding. These results demonstrate that in vitro the Sry $\delta$ protein binds specifically to two sites located between 20 and 40 bp upstream of the presumptive $b c d$ TATA 

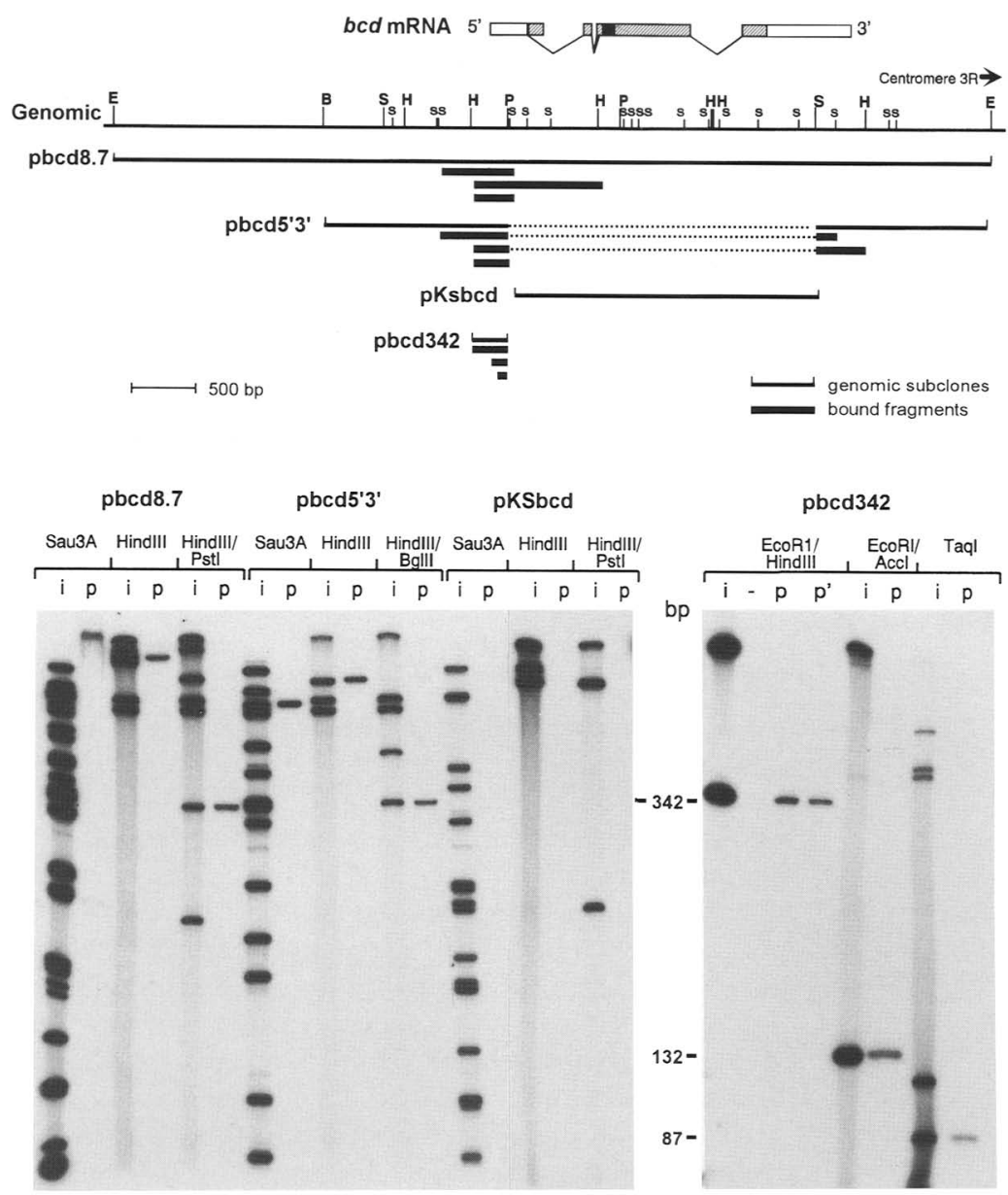

Figure 4. Binding of the Sry $\delta$ protein to the $b c d$ promoter region. (Top) Schematic representation of the $b c d$ genomic region. Restriction sites within the 8.7-kb EcoRI DNA fragment encompassing a functional $b c d$ gene are indicated; $(\mathrm{E})$ EcoRI; (B) BamHI; (S) SalI, (H) HindIII, (s) Sau3AI, (P) PstI. The genomic DNA fragments contained in pbcd 8.7, pcd $5^{\prime} 3^{\prime}$, pksbcd, and pbcd 342 , respectively, are indicated under the restriction map as bracketed lines. Bold lines indicate the immunoprecipitated fragments for each bcd genomic subclone. (Bottom) The different DNAs were cut with the indicated restriction enzyme(s), end-labeled, and immunoprecipitated in presence of the Sry $\delta$ protein and anti-Sry $\delta$ antibody. (i) Input $\mathrm{DNA}_{\text {; }}(\mathrm{p})$ precipitated DNA; $\left(\mathrm{p}^{\prime}\right)$ precipitated DNA using a monoclonal antibody directed against the $\mathrm{T} 7$ tag of the $E$. coli-produced Sry $\delta$ protein; $(-)$ precipitated DNA in the absence of antibody. The 87-bp TaqI-TaqI DNA fragment includes the TaqI-PstI bcd genomic sequence (Fig. 5A) plus 17 bp of linker. box. Together with the dependence of $b c d$ transcription on wild-type sry $\delta$ activity, this finding supports the conclusion that Sry $\delta$ directly controls $b c d$ transcription by binding to a $b c d$ cis-regulatory element.

The Sry $\delta$-binding site is required for wild-type levels of bcd expression

To test the importance of the Sry $\delta$-binding sites for $b c d$ transcription in vivo, we introduced the $\mathrm{Nl}$ mutation in an otherwise intact 8.7-kb bcd genomic fragment within a P-element transformation vector. The wild-type $\mathrm{p}[b$ $c d G E]$ and mutated $\mathrm{p}[b c d G E N 1]$ constructs were introduced into the fly genome, and transgenic Drosophila lines were established. We first stained embryos laid by transformant females with anti-Krüppel antibodies as a measure of bcd activity. Figure $6 \mathrm{~A}$ shows that as the number of $\mathrm{p}[b c d G E]$ copies increases from one to two, the anterior boundary of Krüppel expression shifts to a progressively more posterior position. This reflects increased bcd activity in early embryos as a result of expression of $\mathrm{p}[b c d G E]$, as documented previously in similar experiments by Driever and Nüsslein-Volhard (1988). No shift could be detected in the presence of two copies of $\mathrm{p}[b c d G E N 1]$ (tested with two independent transformed lines, Fig. 6A). These results indicate that the N1 mutation impairs the expression of the $b c d$ transgene. To determine the severity of this reduction in $b c d$ activity the mutated $b c d$ transgene was tested for its ability to rescue the bcd phenotype. One or two copies of $\mathrm{p}[b c d G E]$ or $\mathrm{p}[b c d G E N 1]$ were introduced in females homozygous for the strong $b c d^{6}$ recessive mutation. While one copy of $\mathrm{p}[b c d G E]$ rescues all of the alterations due to the $b c d^{6}$ mutation and allows embryos to develop into fertile adult flies, $\mathrm{p}[b c d G E N 1]$ does not rescue the $b c d$ phenotype. Even with two copies, we still observe a deletion of all the head and thoracic structures and, in most cases, the first or the first two abdominal segments (Fig. $6 \mathrm{~B})$. Therefore, there is no improvement of the $b c d$ phenotype by the $\mathrm{p}[b c d G E N 1]$ construct. Together, the results from both overexpression (Fig. 6A) and phenotypic rescue experiments (Fig. $6 \mathrm{~B}$ ) establish that alteration of one Sry $\delta$-binding site in the $b c d$ promoter region results in a strong decrease of $b c d$ transcription to a level insufficient for significant $b c d$ activity in embryos.

\section{Discussion}

Sry $\delta$, a transcription factor upstream of bcd

A small number $(\sim 30)$ of coordinate genes that are spe- 

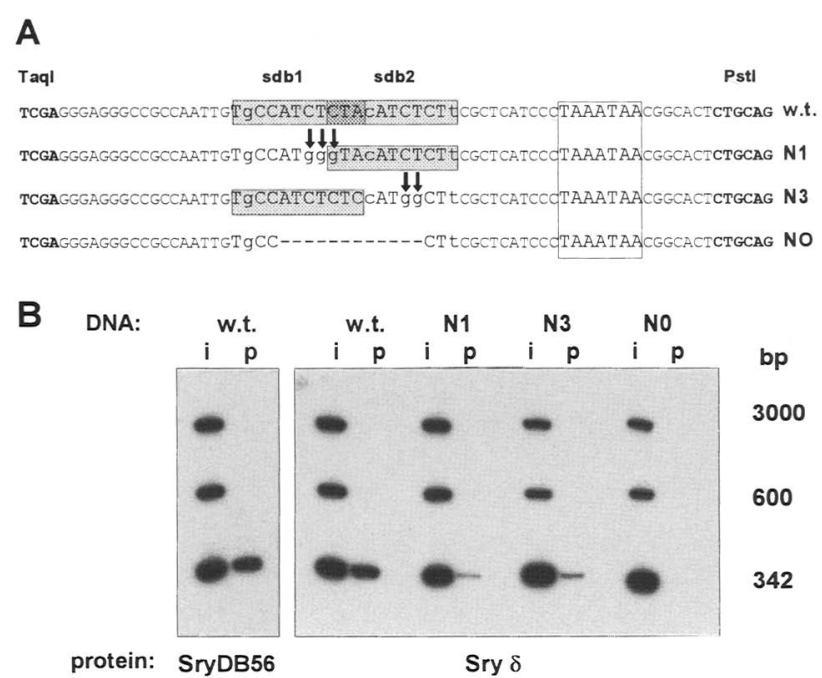

Figure 5. Mutational analysis of the Sry $\delta$-binding site in the $b c d$ promoter. (A) Nucleotide sequence of the TaqI-PstI bcd DNA fragment $(70 \mathrm{bp})$, with the two potential Sry $\delta$-binding sites $(\mathrm{sdb} 1, \mathrm{sdb} 2)$ boxed and shaded. $\mathrm{T} / \mathrm{C} \rightarrow \mathrm{G}$ point mutations were introduced in the core of either binding site ( N1 and N3 mutations) while a 12-nucleotide deletion (NO mutation) removes most of the two binding sites. The presumptive $b c d$ TATA box is boxed. $(B)$ Immunoprecipation analysis of Sry $\delta / b c d$ interaction. The wild-type Sry $\delta$ or the Sry DB56 protein was incubated with restriction enzyme-digested plasmid DNA containing $1.8 \mathrm{~kb}$ of sequence upstream of the $b c d$ transcription start, either wild type or mutated (N1, N3 or NO); (i) Input $\mathrm{DNA}_{;}(\mathrm{p})$ precipitated DNA.

cifically required during oogenesis for the determination of the Drosophila embryonic axes were discovered in genetic screens for maternal-effect mutations (for review, see St Johnson and Nüsslein-Volhard 1992). bcd is the key gene of the anterior system responsible for development of head and thorax. The Bcd protein is distributed in a concentration gradient along the anteroposterior axis of the embryo and is a transcription regulator that is thought to act as a morphogen by eliciting distinct transcriptional responses from its several targets at different concentrations. Thus, one exciting feature of Bcd is its concentration-dependent action (for review, see Driever 1993). Nothing has been reported, however, concerning the transcriptional control of $b c d$ itself. Failure to isolate maternal-effect mutations specifically affecting $b c d$ transcription (contrary to mutations affecting $b c d$ mRNA localization) suggests that factors with more pleiotropic functions are involved. We report here that one specific function of the zygotic-lethal sry $\delta$ gene is to directly control $b c d$ transcription. This sry $\delta$ germ-line function was brought to light by expressing a chimeric Sry $\delta /$ Sry $\beta$ protein in sry $\delta$ mutant females. Progressively more severe anterior pattern defects are observed when going from weak to strong sry $\delta$ mutant alleles, suggesting a progressive reduction of bcd activity. A gradual decrease in $b c d$ mRNA accumulation in ovaries corroborates this embryonic phenotype. This observation suggested that sry $\delta$ positively regulates $b c d$ tran- scription. Because localization of $b c d$ mRNA at the anterior pole of the oocyte is a critical prerequisite for its function, the requirement of sry $\delta$ for $b c d$ mRNA accumulation could, however, also be at the post-transcriptional level. However, this possibility can be ruled out for several reasons. First, phenotypes resulting from $\mathrm{mu}-$ tations in exu, swa, or stau, three maternal genes required for localization of $b c d$ RNA to the anterior end of the egg, show only superficial resemblance to the bcd phenotype (Fronhöfer and Nüsslein-Volhard 1987). Second, the residual $b c d$ mRNA present in weak sry $\delta \mathrm{mu}$ tant ovaries is correctly localized. Finally, it has already been shown that delocalization of $b c d$ mRNA following removal of cis-acting elements within the 3 '-untranslated region does not affect the accumulation level of this mRNA in ovaries (McDonald et al. 1993).

\section{Sry $\delta$ directly controls bed transcription}

If Sry $\delta$ directly controls $b c d$ transcription, it should be possible to identify a $b c d$ cis-acting regulatory sequence binding the Sry $\delta$ protein and mimic the effect of sry $\delta$ mutations by altering this sequence. Two in vitro Sry $\delta$-binding sites are found in a single region that is located in the proximal bcd promoter, $20 \mathrm{bp}$ upstream of the putative TATA box. The sequence of these two overlapping sites is almost identical to the Sry $\delta$ consensus recognition site, as determined previously starting from whole Drosophila genomic DNA (Payre et al. 1991), and in the orientation conferring the most efficient transcriptional activation by Sry $\delta$ in cell transfection assays (F. Payre, P. Buono, and A. Vincent, in prep.). Alteration of one of these Sry $\delta$-binding sites results in a drastic reduction in bcd activity, indicating that this site is critical for $b c d$ expression. The clear phenotypic correlation between the effect of mutations in Sry $\delta$, the alteration of the Sry $\delta$-binding site in $b c d$ DNA, and mutations in $b c d$ itself strongly argues in favor of a positive and direct control of $b c d$ transcription by Sry $\delta$.

Double heterozygote embryos mutant for a strong $b c d$ mutation $\left(b c d^{6}\right)$ and a null for sry $\delta$ are wild type (data not shown). Furthermore, the relative levels of $b c d$ mRNA in flies carrying either two or four copies of $b c d$ are not significantly changed by reducing the dose of wild-type sry $\delta$ from two to one. Therefore, sry $\delta$ activity in ovaries is in excess relative to that required for normal $b c d$ transcription. Yet, $b c d$ appears to be highly sensitive to mutations in sry $\delta$, indicating that the cis-acting element binding Sry $\delta$ in the $b c d$ promoter is critical for $b c d$ transcription, as subsequently confirmed by the in vivo effect of mutating this binding site.

Genetic experiments in which not only the overall level, but also the shape, of the Bcd gradient is modified have revealed that at least two different regions of the embryonic fate map are specified in a Bcd concentration-dependent manner (Driever and Nüsslein-Volhard 1988 b; for review, see Driever 1992). Low concentrations of Bcd autonomously define thorax identity, whereas high levels define head. Correlation between the calculated relative levels of $b c d$ mRNA in various sry $\delta \mathrm{mu}-$ 

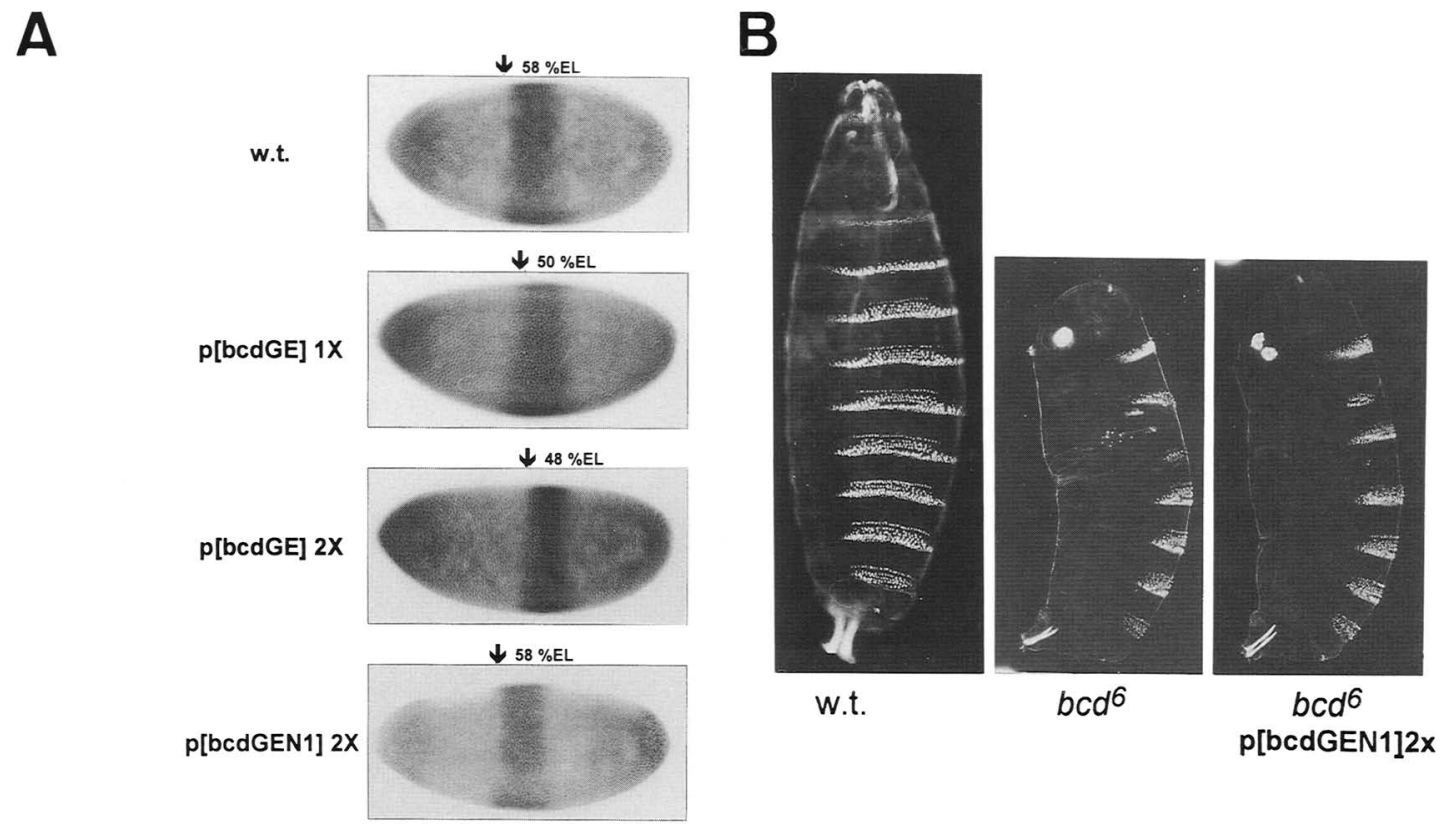

Figure 6. Effect of the N1 promoter mutation on bcd activity in early embryos. (A) Anti-Krüppel immunostaining of whole-mount embryos from females either wild type (w.t.) or carrying one or two copies of $\mathrm{p}[b c d G E]$ construct. Position of the anterior boundary of Krüppel expression was measured with camera lucida examination of $\sim 55$ blastoderm-stage embryos of each genotype. Wild-type position is at $58 \%$ egg length $(E L)$, S.D. $=1.02 ; \mathrm{p}[b c d G E] 1 \times$ at $50.3 \% \mathrm{EL}$, S.D. $=1.07$; p $[b c d G E] 2 \times$ at $47.7 \%$ EL, S.D. $=1.3 ; \mathrm{p}[b c d G E N 1] 2 \times$ at $57.9 \%$ EL, S.D. $=1.2$. The same value $\{$ S.D. $=0.97$ ) was obtained with a second $\mathrm{p}[b c d G E N 1]$ strain. $(B)$ Cuticle preparations of embryos from either wild-type females or females homozygous for the $b c d^{6}$ mutation with or without two copies of the p[bcdGEN1] transgene. Note that whereas one copy of $\mathrm{p}[b c d G E]$ is able to restore normal segmentation and hatching (Berleth et al. 1988; data not shown) there is no significant rescue of the $b c d$ phenotype by two copies of $\mathrm{p}[b c d G E N 1]$. Insertions on the first or second chromosome were crossed into a $b c d$ mutant background to give stocks of the following genotype $\mathrm{p}\left[w^{+}, b c d G E\right.$ or $\left.b c d G E N 1\right] / C y O$ or $F M 7 ; b c d^{6} / T M 2$. These stocks were used to generate flies homozygous for $b c d^{6}$ and either heterozygous $(1 \times)$ or homozygous $(2 \times)$ for the transposon.

tant combinations and the severity of the resulting $b c d$ phenotype suggest that the level of $b c d$ expression required for complete head formation must be $>30 \%$ of wild type level.

\section{The bcd regulatory function of Sry $\delta$ revealed by $a$ designed finger swap}

The crystal structure of the five finger GLI-DNA complex has brought new insights on $\mathrm{C} 2 \mathrm{H} 2$ zinc fingerDNA recognition by showing that individual fingers may contribute from 0 to 5 direct nucleotide contacts and/or make extensive protein interactions with other fingers (Pavletich and Pabo 1993). Furthermore, systematic disruption of each of the nine zinc fingers of TFIIIA has suggested structurally and functionally nonuniform interactions of zinc fingers with DNA (Del Rio et al. 1993; Rolling et al. 1993). The Sry DB56 protein was designed to test for the in vivo consequences of the selective exchange of a subset of fingers between Sry $\delta$ and its close paralog Sry $\beta$ (Payre et al. 1990; Ferrer et al. 1994). This finger swap leads to only minor changes in DNA recognition properties in vitro, as Sry DB56 binds to Sry $\delta$-binding sites, both consensus or within $b c d$, with an affinity similar to the wild-type protein. Sry DB56 is also able to substitute for Sry $\delta$ in most, if not all, of its functions throughout development, except $b c d$ transcription. Therefore, and despite the fact that exchanging fingers 5 and 6 introduces 33 amino acid changes, Sry DB56 behaves as a sry $\delta$ site-specific allele. It contrasts with the pleiotropic, yet in part allele specific, phenotypes associated with different single amino acid changes in the third Sry $\delta$ finger (Crozatier et al. 1992). Specific failure of Sry DB56 to promote $b c d$ transcription could be attributable to either inefficient binding to the $b c d$ promoter in vivo or improper proteinprotein interactions involved in $b c d$ transcription these two possibilities not being mutually exclusive. In this regard, it is interesting to note the peculiar twin structure of the Sry $\delta$-binding sites in the $b c d$ promoter, with the two sites partly overlapping. Our data on Sry DB56 illustrate further the functional versatility of $\mathrm{C} 2 \mathrm{H} 2$ zinc finger motifs in promoter specificity and modulation of RNA polymerase II transcription.

\section{Zygotic factors and maternal programs}

Why was sry $\delta$ not identified in previous genetic screens for genes involved in establishing the coordinates of the embryo? While a number of arguments suggest that most maternal genes specifically involved in establishing positional information in the egg have been identified, the 
screens have not been designed to identify maternal effect genes with zygotic functions. Systematic germ-line clonal analysis of $\mathrm{X}$-linked zygotic lethal mutations has identified numerous additional genes that affect specific aspects of embryonic development (Perrimon et al. 1989 ). In this study $27 \%$ of larval-pupal lethal mutations on the $\mathrm{X}$ chromosome were found to be germ-line cell lethals. Germ-line clones for sry $\delta$ mutations produce small eggs with severe defects ranging from an abnormal anterior end and fused dorsal appendages, to a more drastic open chorion phenotype (Schüpbach and Wieschaus 1991), and in no case do the few eggs laid develop (M. Crozatier, unpubl.). Although this phenotype still deserves a more thorough analysis, it already indicates that failure to express bcd correctly is only one specific consequence of the effect of sry $\delta$ mutations, that could not be recognized in a classic germ-line clonal analysis. Requirement for sry $\delta$ function at multiple developmental steps, including oogenesis, probably conceals its specific role in the control of $b c d$ transcription. Whereas the various somatic and germ-line phenotypes associated with sry $\delta$ mutations correlate with the widespread expression of Sry $\delta$ throughout development, they contrast with the temporal restriction of $b c d$ expression. Requirement of Sry $\delta$ for $b c d$ transcription underscores the fundamental role of ubiquitously expressed transcription factors in regulating the expression of specific genes and achieving specific developmental programs. It also sheds new light on the molecular dialog between zygotic factors and maternal genes in building a proper Drosophila egg.

\section{Materials and methods}

\section{Fly strains}

The four sry $\delta$ ethylmethane sulfonate (EMS)-induced alleles sry $\delta^{12}$, sry $\delta^{14}$, sry $\delta^{S F 1}$, sry $\delta^{S F 2}$ and the $D f(3 R) X 3 F(D f X 3 F)$ deficiency strains were described in Crozatier et al. (1992). The $b c d^{(E 1)}$ mutant strain was obtained from the Tübingen Stock Center (Tearle and Nüsslein-Volhard 1987). All mutant alleles were kept out as balanced stocks. P-element-mediated transformation was done as described in Spradling and Rubin (1982) using the helper plasmid $\mathrm{p} \pi 25.7$ w.c. as a source of transposase. The $r y^{506}$ strain was used as wild-type strain for RNA preparations, and for injection of the $\mathrm{p}\left[\right.$ sry $\left.B D 56, r y^{+}\right]$rescue construct. The $\mathrm{p}[b c d G E]$ and $\mathrm{p}[b c d G E N 1]$ constructs were injected into a white $\left(w^{-}\right)$strain. Flies were grown under standard conditions at $25^{\circ} \mathrm{C}$, and eggs processed for the determination of the cuticular phenotypes as described (Wieschaus and Nüsslein-Volhard 1986).

\section{Generation of sry $\delta^{-}$homozygous germ-line clones}

Crosses were conducted to produce flies trans-heterozygous for a sry $\delta$ mutation and $\mathrm{p}\left[0 \mathrm{O}^{D 1}-8\right]$ (Mevel-Ninio et al. 1994). Because sry $\delta$ is recessive, these flies are phenotypically sry $\delta^{+}$but totally lack developed ovaries, owing to the effect of the ovo ${ }^{D 1}$ dominant female sterile mutation. Following X-ray treatment (dose 1000 rads) during the first-instar larval stage to induce mitotic recombination, high frequency germ-line clones of cells homozygous for sry $\delta$ that lack the dominant female sterile mutation were obtained (in our hands, in $\sim 20 \%$ of treated flies). Female flies were dissected between 3 and 20 days after eclos- ing, and ovarian morphology was examined using DIC optics and fluorescent probes for actin and DNA. The few laid embryos were collected and stained with anti-Bcd antibody.

General molecular techniques and $D N A /$ protein-binding assays

RNA preparation from ovaries and Northern blot analysis were done according to Vincent et al. (1984), using random primed probes. Expression in E. coli and purification of the recombinant Sry $\beta$, Sry $\delta$, and Sry DB56 proteins, EMSA, and immunoprecipitation procedures were as described in Payre and Vincent (1991). A SacI fragment (positions 4448-5504 from pXDB56, see below) was used to replace zinc fingers 5-6 of Sry $\delta$ by those of Sry $\beta$ in the $E$. coli production vector pSDA1 (Payre et al. 1989). The genomic $8.7-\mathrm{kb}$ EcoRI fragments containing the functional bcd gene (Berleth et al. 1988) and a fragment containing only the $5^{\prime}$ and $3^{\prime} b c d$ nontranscribed regions were prepared from the P20 transformation vector (a gift from W. Driever, Massachusetts General Hospital, Boston) and the Casper bcd BgIII (a gift from C. Desplan, Rockefeller University, NY), respectively, and cloned into the pGEM7ZP vector (Promega). pksbcd was obtained from C. Desplan; pbcd 342 was constructed by inserting the $b c d$ genomic region $(+869$ to +1241$)$ as a HindIII-Pst I fragment in PGEM7ZP. For site-specific mutagenesis the procedure of Kunkel (1985) was used along with modification of some steps to optimize production of high quality uracylated single stranded DNA. Sequences of the oligonucleotides used to introduce the N1, N3, and N0 mutations in the pbcd1.8 subclone [1.8-kb BamHI-PstI $\mid+1241\}$ bcd fragment in pTZ 18R] are N1 5'-CAATTGTGCCATGGGTACATCTCTTCGC-3'; N3, 5'CCATCTCTCCATGGCGCTCATCCC-3'; and No, 5'-GCCGCCAATTGTGCCCTTCGCTCATCCC- $3^{\prime}$. N1 and N3 oligonucleotides incorporated a $\mathrm{NcoI}$ restriction site (underlined) to facilitate mutant identification. The specific nature of the introduced mutations was then confirmed by sequencing.

\section{Transformation constructs and transgenic fly strains}

$p$ (sry DB56) construct The DNA fragment encoding fingers 5 and 6 of Sry $\beta$ was prepared from a sry $\beta$ genomic clone as a NdeI-SmaI fragment, position 1494-1663/the numbering referring to the sry sequence in Vincent et al. 1985). The NdeI and SmaI sites were created by site-directed mutagenesis using the oligonucleotides 5'-GAACGCCCTCATTATGCGAGGTGTG CC-3' and 5'-CGAGAAGCCCGGGCGGCGGAGGCC-3', respectively. This sry $\beta$ fragment was then inserted into a genomic clone containing the functional sry $\delta$ gene (from an $X b a I$ site, position 2486 to an HindIII site, position 6074) (Crozatier et al. 1992) in replacement of the sry $\delta \mathrm{NdeI-SmaI} \mathrm{fragment} \mathrm{(po-}$ sition 5171-5340). The SmaI site was created in the sry $\delta$ sequence, using the 5'-CGCGAACCCCGGGAACGACCTCGCCC-3' oligonucleotide. This exchange of fragments results in the selective replacement of fingers 5 and 6 of Sry $\delta$ by the corresponding fingers of Sry $\beta$ (plasmid pXDB56). The chimeric sry $\delta /$ sry $\beta$ (sry DB56) gene was then inserted into the $\left[r y^{+}\right]$ P-element transformation vector $\mathrm{pDm} 23$.

$p$ /bcdGE $/$ and $p$ /bcdGEN1] constructs After introduction of the N1 mutation, the 1.8-kb BamHI-PstI bcd genomic fragment was put back into $\mathrm{p}[\mathrm{bcdGE}]\left[\mathrm{a} \mathrm{w}^{+} \mathrm{P}\right.$-vector containing the 8.7 $\mathrm{kb} b c d$ genomic fragment obtained from C. Nüsslein-Volhard (Max Planck Institute, Tübingen, Germany) as CasgbcdE] to give the $\mathrm{p}[b c d G E N 1]$ construct. 
In situ hybridization and antibody staining

Whole-mount ovaries were dissected in EBR solution (130 mM $\mathrm{NaCl}, 5 \mathrm{~mm} \mathrm{KCl}, 2 \mathrm{~mm} \mathrm{CaCl}_{2}, 10 \mathrm{~mm}$ HEPES at $\mathrm{pH} \mathrm{6.9)} \mathrm{and}$ hybridized with digoxigenin-labeled DNA probes, prepared with the genius kit from Boehringer Mannheim, using the procedure described by Tautz and Pfeifle (1989), with minor modifications (Ephrussi et al. 1991). Whole-mount immunodetection on early embryos was as described by Payre et al. (1990), using a polyclonal anti-Krüppel antibody raised in rabbits (a gift from M. Levine's laboratory, University of California, San Diego), or a monoclonal anti-Bcd antibody obtained from C. Nüsslein-Volhard.

\section{Acknowledgments}

We are grateful to the Tübingen Drosophila Stock Center for sending us mutant strains, W. Driever and C. Nüsslein-Volhard for bcd DNA clones and antibody, A. Ephrussi for the osk cDNA, G. Struhl for the bcd $4 \times$ transformed strain, and M. Mevel-Ninio for the $\mathrm{p}[\mathrm{ovoD} 1 / 8]$ strain and sharing results prior to publication. We thank C. Desplan, H. Jäckle, and J. Smith for their comments on the manuscript and our colleagues at Toulouse for suggestions and discussions throughout the course of this work. The work was supported by CNRS, Institut National de la Santé et de la Recherche Médicale (INSERM) (grant 930112), Association pour la Recherche sur le Cancer and Région Midi-Pyrénées.

The publication costs of this article were defrayed in part by payment of page charges. This article must therefore be hereby marked "advertisement" in accordance with 18 USC section 1734 solely to indicate this fact.

\section{References}

Berleth, T., M. Burri, G. Thoma, D. Bopp, S. Richstein, G. Frigerio, M. Noll, and C. Nüsslein-Volhard. 1988. The role of localization of bicoid RNA in organizing the anterior pattern of the Drosophila embryo. EMBO J. 7: 1749-1756.

Crozatier, M., K. Kongsuwan, P. Ferrer, J. Merriam, J. Lengyel, and A. Vincent. 1992. Single amino-acid changes in separate domains of the Drosophila sry $\delta$ zinc finger protein cause embryonic and sex-biased lethality. Genetics 131: 905-916.

Del Rio, S., S.R. Menzes, and D.R. Setzer. 1993. The function of individual zinc fingers in sequence-specific DNA recognition by transcription factor IIIA. I. Mol. Biol. 233: 567-579.

Driever, W. 1992. The bicoid morphogen: Concentration dependent transcriptional activation of zygotic target genes during early Drosophila development. In Transcriptional regulation (ed. S.L. Mc Knight and K.R. Yamamoto), pp. 12211250. Cold Spring Harbor Laboratory Press, Cold Spring Harbor, New York.

Driever, W. and C. Nüsslein-Volhard. 1988a. A gradient of bicoid protein in Drosophila embryos. Cell 54: 83-93.

— 1988b. The bicoid protein determines position in the Drosophila embryo in a concentration-dependent manner. Cell 54: 95-104.

- 1989. The bicoid protein is a positive regulator of hunchback transcription in the early Drosophila embryo. Nature 337: 138-143.

Driever, W., V. Siegel, and C. Nüsslein-Volhard. 1990. Autonomous determination of anterior structures in the early Drosophila embryo by the bicoid morphogen. Development 109: 811-820.

Ephrussi, A., L.K. Dickinson, and R. Lehmann. 1991. Oskar organises the germ plasm and directs localization of the posterior determinant nanos. Cell 66: 37-50.

Ferrer, P., M. Crozatier, C. Salles, and A. Vincent. 1994. Interspecific comparison of Drosophila serendipity $\delta$ and $\beta$; multimodular structure of these $\mathrm{C} 2 \mathrm{H} 2$ zinc finger proteins. $J$. Mol. Evol. 38: 263-273.

Finkelstein, R. and N. Perrimon. 1990. The orthodenticle gene is regulated by bicoid and torso and specificies Drosophila head development. Nature 346: 485-488.

Frohnhöfer, H.G. and C. Nüsslein-Volhard. 1986. Organization of anterior pattern in the Drosophila embryo by the maternal gene bicoid. Nature 324: 120-125.

- 1987. Maternal genes required for the anterior localization of bicoid activity in the embryo of Drosophila. Genes \& Dev. 1: 880-890.

Hoch, M. and H. Jäckle. 1993. Transcriptional regulation and spatial patterning in Drosophila. Cur. Opin. Genet. Dev. 3: 566-573.

Hoch, M., E. Seifert, and J. Jäckle. 1991. Gene expression mediated by cis-regulatory sequences of the Krüppel gene in response to the anterior morphogens bicoid and hunchback. EMBO J. 10: 2267-2278.

Kunkel, T.A. 1985. Rapid and efficient site-specific mutagenesis without phenotypic selection. Proc. Natl. Acad. Sci. 82: 488-492.

McDonald, P.M., K. Kerr, J. Smith, and A. Leask. 1993. RNA regulatory element BLEl directs the early steps of bicoid mRNA localization. Development 118: 1233-1243.

Mevel-Ninio, M., I. Guénal, and B. Limbourg-Bouchon. 1994. Production of dominant female sterility in Drosophila melanogaster by insertion of the ovoD1 allele on autosomes: Use of transformed strains to generate germ line mosaics. Mech. Dev. 45: 155-162.

Noselli, S. and A. Vincent. 1992. The Drosophila melanogaster ribosomal protein $L 17 A$-encoding gene. Gene 118: 273-278.

Noselli, S., F. Payre, and A. Vincent. 1992. Zinc fingers and other domains cooperate in binding of the Drosophila sry $\beta$ and $\delta$ proteins at specific chromosomal sites. Mol. Cell. Biol. 12: 724-733.

Nüsslein-Volhard, C., G. Fronhöfer, and R. Lehmann. 1987. Determination of anteroposterior polarity in Drosophila. Science 238: 1675-1681.

Pavletich, N.P. and C.O. Pabo. 1993. Crystal structure of a fivefinger GLI-DNA complex: New perspectives on zinc fingers. Science 261: 1701-1707.

Payre, F. and A. Vincent. 1991. Genomic targets of the sry $\beta$ and sry $\delta$ zinc finger proteins and their respective DNA recognition sites. EMBO I. 10: 2533-2541.

Payre F., C. Yanicostas, and A. Vincent. 1989. Serendipity $\delta$, a DNA-binding finger protein associated with embryonic nuclei at the onset of zygotic gene transcription. Dev. Biol. 136: 469-480.

Payre, F., S. Noselli, V. Lefrére, and A. Vincent. 1990. The closely related Drosophila sry $\beta$ and sry $\delta$ zinc finger proteins show differential embryonic expression and distinct patterns of binding sites on polytene chromosomes. Development 110: 141-145.

Perrimon, N., L. Engstrom, and A.P. Mahowald. 1989. Zygotic lethals with specific maternal effect phenotypes in Drosophila melanogaster. I Loci on the X chromosome. Genetics 121: 333-352.

Pignoni, F., E. Steingrimsson, and J.A. Lengyel. 1992. bicoid and the terminal system activate tailless expression in the early Drosophila embryo. Development 115: 239-251.

Rollins, M.B., S. Del Rio, A.L. Galey, D.R. Setzer, and M.T. Andrews. 1993. Role of TFIIIA zinc fingers in vivo: Analysis 
Payre et al.

of single-finger in Developing Xenopus embryos. Mol. Cell. Biol. 13: 4776-4783.

Ronchi, E., J. Treisman, N. Dostatni, G. Struhl, and C. Desplan. 1993. Down-regulation of the Drosophila morphogen bicoid by the torso receptor-mediated signal transduction cascade. Cell 74: 347-355.

Rubin, G. and A. Spradling. 1992. Genetic transformation of Drosophila with transposable element vectors. Science 218: 348-353.

Schüpbach, T. and E. Wieschaus. 1986. Maternal-effect mutations altering the anterior-posterior pattern of the Drosophila embryo. Wilhelm Roux's Arch. Dev. Biol. 195: 302317.

- 1991. Female sterile mutations on the second chromosome of Drosophila melanogaster II. Mutations blocking oogenesis or altering egg morphology. Genetics 129: 11191136.

St Johnston, D. and C. Nüsslein-Volhard. 1992. The origin of pattern and polarity in the Drosophila embryo. Cell 68: 201219.

St Johnston, D., W. Driever, T. Berleth, S. Richstein, and C. Nüsslein-Volhard. 1989. Multiple steps in the localization of bicoid RNA to the anterior pole of the Drosophila oocyte. Development suppl. 13-19.

Struhl, G., K. Struhl, and P.M. Macdonald. 1989. The gradient morphogen bicoid is a concentration-dependent transcriptional activator. Cell 57: 1259-1273.

Tautz, D. 1988. Regulation of the Drosophila segmentation gene hunchback by two maternal morphogenetic centers. Nature 332: 281-384.

Tautz, D. and C. Pfeifle. 1989. A nonradioactive in situ hybridization method for the localization of specific RNAs in Drosophila embryos reveals a translational control of the segmentation gene hunchback. Chromosoma 98: 81-85.

Tearle, R. and C. Nüsslein-Volhard. 1987. Tübingen mutants and stocklist. Dros. Inf. Serv. 66: 209-269.

Theurkauf, W.E., S. Smilery, M.L. Wong, and B.M. Alberts. 1992. Reorganization of the cytoskeleton during Drosophila oogenesis; implications for axis specification and intracellular transport. Development 115: 923-936.

Vincent, A., P. O'Connell, M. Gray, and M. Rosbash. 1984. Drosophila maternal and embryo mRNAs transcribed from a single transcription unit use alternate combinations of exons. $E M B O$ I. 3: 1003-1013.

Vincent, A., H. Colot, and M. Rosbash. 1985. Sequence and structure of the serendipity locus of Drosophila melanogaster: A densely transcribed region including a blastoderm specific gene. I. Mol. Biol. 186: 149-166.

Wang, C. and R. Lehmann. 1991. Nanos is the localized posterior determinant in Drosophila. Cell 66: 637-647.

Wieschaus, E. and C. Nüsslein-Volhard. 1986. Looking at embryos. In Drosophila: A practical approach (ed. D.B. Roberts), pp 199-227. IRL Press, Washington, D.C. 


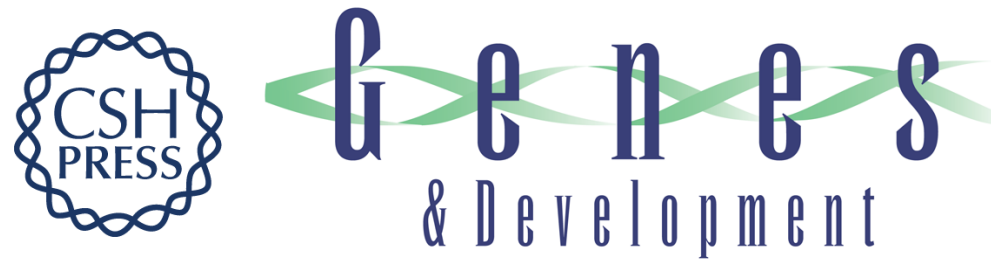

\section{Direct control of transcription of the Drosophila morphogen bicoid by the serendipity delta zinc finger protein, as revealed by in vivo analysis of a finger swap.}

F Payre, M Crozatier and A Vincent

Genes Dev. 1994, 8:

Access the most recent version at doi:10.1101/gad.8.22.2718

References This article cites 40 articles, 14 of which can be accessed free at:

http://genesdev.cshlp.org/content/8/22/2718.full.html\#ref-list-1

License

Email Alerting

Service

Receive free email alerts when new articles cite this article - sign up in the box at the top right corner of the article or click here.

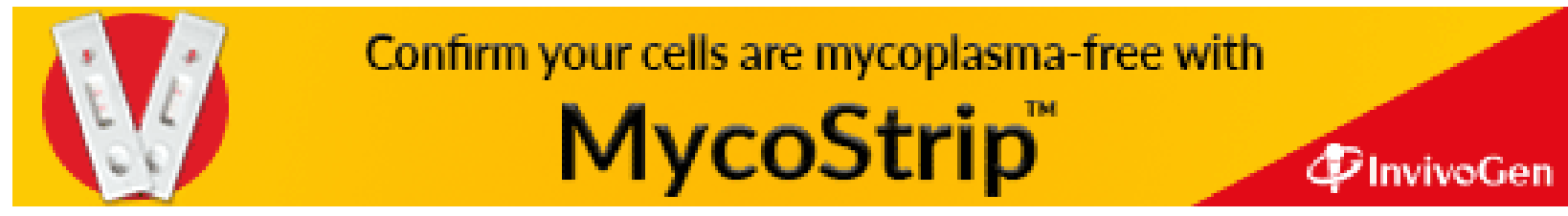

